\title{
Possibility of magnetospheric VLF response to atmospheric infrasonic waves
}

\author{
P. A. Bespalov ${ }^{1}$ and O. N. Savina ${ }^{2,3}$ \\ ${ }^{1}$ Institute of Applied Physics, Russian Academy of Sciences, 46 Ulyanov St, 603950 Nizhny Novgorod, Russia \\ ${ }^{2}$ State University the Higher Scool of Economics, 25/12 B. Pecherskaya St, 603155 Nizhny Novgorod, Russia \\ ${ }^{3}$ State Technical University, 24 Minina St, 603600 Nizhny Novgorod, Russia \\ (Received May 31, 2010; Revised March 24, 2011; Accepted May 6, 2011; Online published July 27, 2012)
}

\begin{abstract}
In this paper, we consider a model of the influence of atmospheric infrasonic waves on VLF magnetospheric whistler wave excitation. This excitation occurs as a result of a succession of processes: a modulation of the plasma density by acoustic-gravity waves in the ionosphere, a reflection of the whistlers by ionosphere modulation, and a modification of whistler wave generation in the magnetospheric resonator. A variation of the magnetospheric resonator $Q$-factor has an influence on the operation of the plasma magnetospheric maser, where the active substances are radiation belt particles, and the working modes are electromagnetic whistler waves. The magnetospheric maser is an oscillating system which can be responsible for the excitation of self-oscillations. These self-oscillations are frequently characterized by alternating stages of accumulation and precipitation of energetic particles into the ionosphere during a pulse of whistler emissions. Numerical and analytical investigations of the response of self-oscillations to harmonic oscillations of the whistler reflection coefficient shows that even a small modulation rate can significantly change magnetospheric VLF emissions. Our results can explain the causes of the modulation of energetic electron fluxes and whistler wave intensity with a time scale from 10 to $150 \mathrm{~s}$ in the day-side magnetosphere. Such quasi-periodic VLF emissions are often observed in the sub-auroral and auroral magnetosphere and have a noticeable effect on the formation of space weather phenomena.
\end{abstract}

Key words: Magnetosphere-ionosphere interactions, wave propagation, wave-particle interactions.

\section{Introduction}

There have been many studies of the effects of processes in space on the ionosphere and atmosphere. Examples include tidal effects, energetic particle precipitation, the generation of acoustic-gravity waves by current systems, effects of the current systems on geomagnetically-induced currents (GIC), and the influence of radiation on the chemistry of the ionosphere. The influence of atmospheric processes on the magnetosphere has received much less attention in the literature. Previous studies have discussed the electromagnetic radiation of lightning discharges, and there are indications of magnetospheric manifestations of earthquakes.

In this paper, we focus on the possible influence of atmospheric acoustic-gravity waves on magnetospheric processes. There are several powerful natural sources of acoustic-gravity waves including, for example, lightning discharges in the atmosphere, and volcanic activity. Acoustic-gravity waves in the ionosphere can cause modulation of the electron plasma density; this modulation can cause several magnetospheric effects:

- Firstly, acoustic-gravity waves can change the reflection coefficient of electromagnetic VLF waves from the ionosphere.

Copyright (c) The Society of Geomagnetism and Earth, Planetary and Space Sciences (SGEPSS); The Seismological Society of Japan; The Volcanological Society of Japan; The Geodetic Society of Japan; The Japanese Society for Planetary Sciences; TERRAPUB.

doi:10.5047/eps.2011.05.024
- Secondly, in the vicinity of intensive ionospheric currents, these variable currents will emit MHD waves at the acoustic-gravity wave frequency.

- Thirdly, and possibly most importantly for our purposes, intensive fluxes of energetic particle precipitation from the magnetosphere to the ionosphere are connected with acoustic-gravity waves.

- Finally, a linear transformation of atmospheric waves into magnetospheric waves is possible in principle. The frequencies and wave vectors of the two types of disturbances must coincide at the appropriate height for this process to occur.

In this paper, we will examine in more detail the first of these possibilities: the modulation of the reflection coefficient of electromagnetic VLF waves from the ionosphere (cf. Bespalov et al., 2003). The reflection coefficient of whistler waves from the ionosphere is determined by several factors, which can be stimulated by acoustic-gravity waves:

- electron density in the ionosphere;

- electronic density gradient;

- small-scale instabilities.

The reflection coefficient from the ionosphere will determine the magnetospheric resonator quality for VLF waves in many respects. The magnetosphere forms a plasma magnetospheric maser in the region of the electron radiation belts. This system is very sensitive even to small modu- 


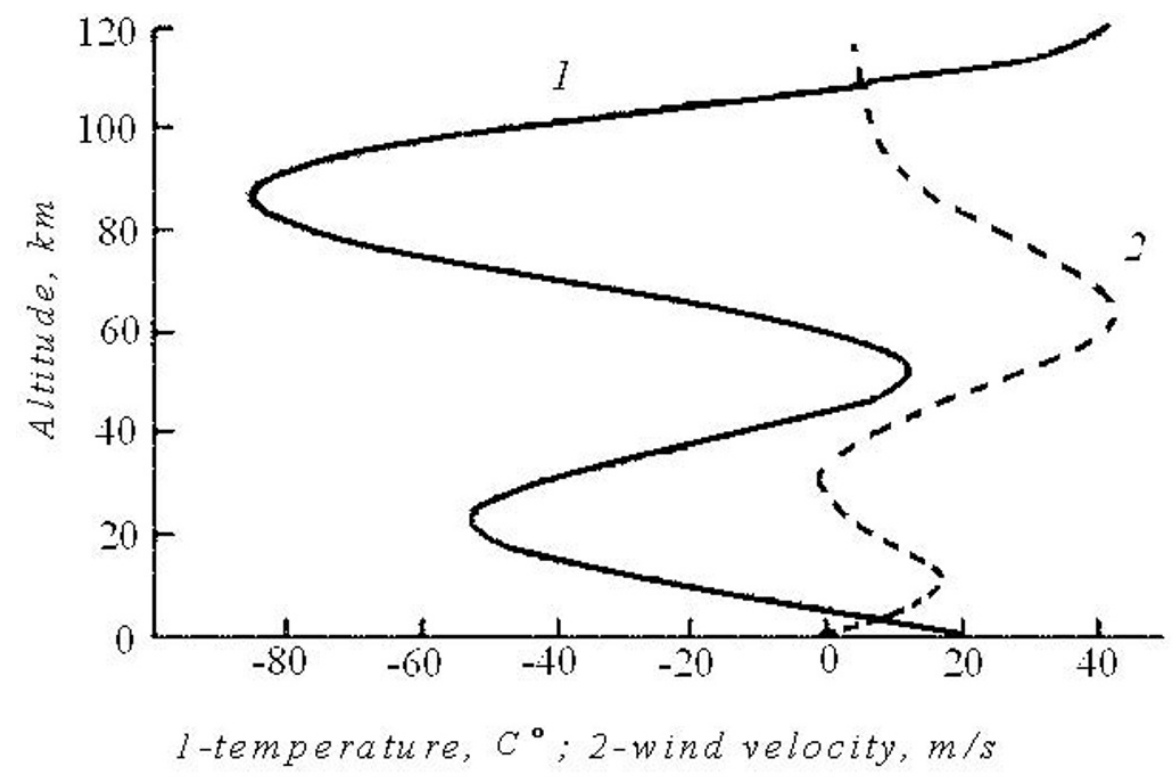

Fig. 1. The altitude profiles of the temperature and horizontal wind velocities used in the calculations.

lations of the magnetospheric resonator quality ( $Q$-factor). We will try to explain this by the calculations below. The plasma magnetospheric maser is especially sensitive to external periodic actions at frequencies close to its natural frequency. From our previous analysis, it follows that the parameters of the system can produce slowly-decaying oscillations representing alternating stages of accumulation of energetic electrons and their precipitation into the ionosphere during pulses of electromagnetic radiation. Oscillations near a steady state with frequency $\Omega_{J}$ and their rate of decay $v_{J}$ are determined by the simple expressions (Bespalov and Trakhtengerts, 1986):

$$
\Omega_{J}=\left(\frac{v}{T_{l}}\right)^{1 / 2}, \quad v_{J}=\frac{1}{T_{l}}
$$

Here, $v$ is the average rate of decay of whistler waves in the magnetospheric resonator, $T_{l}$ is the mean life time of energetic electrons in the magnetic trap, taking into account all factors. For typical conditions in the pre- and post-noon local time sectors of the Earth's magnetosphere, the period of these oscillations $\left(T_{J}\right)$ is between 10 and $150 \mathrm{~s}$, and the $Q_{J}$-factor of the oscillations $\left(Q_{J}=\Omega_{J} / 2 \nu_{J}\right)$ is of the order of several tens in the day-side magnetosphere. Such a high quality factor $Q_{J}$ defines the resonant response of the radiation belts to external effects. Let us note that the oscillations of Eq. (1) can actually be excited by different external actions. For example, in the magnetosphere of Jupiter such oscillations are excited by the daily rotation of the planet (Bespalov and Savina, 2005; Bespalov et al., 2005).

The frequency $\Omega_{J}$ is that of the atmospheric infrasonic wave. Therefore, it is natural to begin our analysis with the study of infrasound propagation in the Earth's atmosphere. We take the infrasound source to be on the Earth's surface.

\section{The Propagation of Infrasonic Waves in the Atmosphere from a Ground-Based Source}

Acoustic-gravity waves occur as a result of the natural processes in the atmosphere discussed above. These waves extend to ionospheric altitudes and can stimulate disturbances of ionospheric plasma (Artru et al., 2001). For a description of the properties of wave perturbations with vertical scales of the order of the changes of the background atmospheric parameters, or greater, it is assumed that the use of models for wave-characteristics calculations is adequate. In this paper, we are interested in disturbances corresponding to low-frequency infrasonic waves, for which the non-isothermicity of the atmosphere is important. If we use a method, based on the analysis of the nonlinear Rikkati equation for the polarization relationship for infrasonic waves, then the calculation of the fields of such waves is substantially simplified. This examination enables a comparatively simple solution to a traditionally complex question about the boundary condition at high altitudes (Savina, 1996). We start from the usual two-dimensional linearized set of atmospheric gas-dynamics equations (Gossard and Hooke, 1975):

$$
\begin{aligned}
& \frac{\partial v}{\partial t}+u_{0} \frac{\partial v}{\partial x}+\frac{1}{\rho_{0}} \frac{\partial p}{\partial y}-v_{\mathrm{k}} \Delta v=0, \\
& \frac{\partial w}{\partial t}+u_{0} \frac{\partial w}{\partial x}+\frac{1}{\rho_{0}} \frac{\partial p}{\partial z}-g \frac{\rho}{\rho_{0}}-v_{\mathrm{k}} \Delta w=0 \\
& \frac{\partial \rho}{\partial t}+u_{0} \frac{\partial \rho_{0}}{\partial x}+w \frac{d \rho_{0}}{d z}+\rho_{0} \frac{\partial u}{\partial x}+\rho_{0} \frac{\partial v}{\partial y}+\rho_{0} \frac{\partial w}{\partial z}=0 \\
& \frac{\partial p}{\partial t}+u_{0} \frac{\partial p}{\partial x}+w \frac{d p_{0}}{d z}=c_{\mathrm{s}}^{2}\left(\frac{\partial \rho}{\partial t}+w \frac{d \rho_{0}}{d z}\right) .
\end{aligned}
$$

Here, $g$ is the acceleration due to gravity; $x$ and $y$ are the horizontal co-ordinates; $z$ is the vertical co-ordinate; $\rho_{0}(z)$ is the unperturbed density of the atmosphere; $\rho$ and $p$ are small perturbations of density and pressure; $w, v$ and $u$ are vertical and horizontal components of the gas velocity; $c_{\mathrm{s}}$ is 


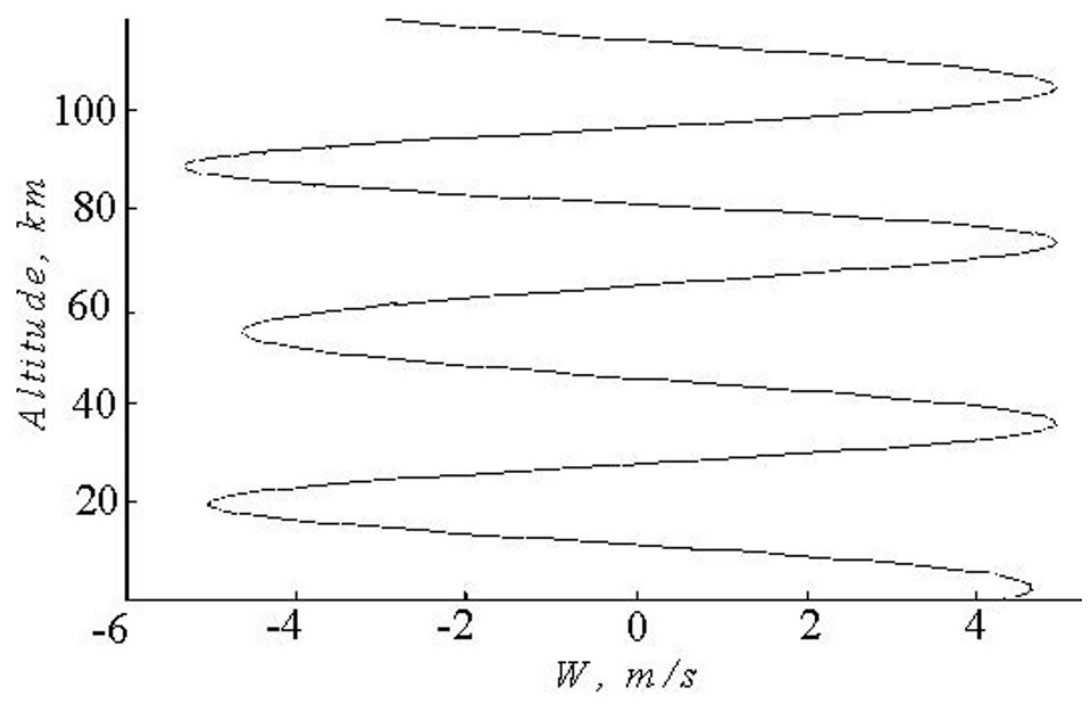

Fig. 2. The altitude profile of calculated $W(z)$.

the sound velocity; $u_{0}$ is wind velocity, which we assume is directed along the $x$-axis and depends only on the vertical coordinate (see Fig. 1), and $v_{\mathrm{k}}$ is the kinematic viscosity.

Let us introduce into the examination a new function:

$$
\Phi\left(\omega, k_{x}, k_{y}, z\right)=-\frac{i \rho_{00} p}{\rho_{0} w Z_{0}} .
$$

Then, from the system of Eq. (2), for a heterogeneous plane wave with frequency $\Omega$ and horizontal wave vector components $k_{x}, k_{y}$ we obtain (Rapoport et al., 2004; Savina et al., 2006):

$$
\begin{aligned}
\frac{\partial \Phi\left(\Omega, k_{x}, k_{y}, z\right)}{\partial z}= & i K\left(1-\Phi^{2}\left(\Omega, k_{x}, k_{y}, z\right)\right) \\
& -2 \chi \Phi\left(\Omega, k_{x}, k_{y}, z\right)
\end{aligned}
$$

$$
\begin{aligned}
\frac{\partial W\left(\Omega, k_{x}, k_{y}, z\right)}{\partial z}= & \left(i K \Phi\left(\Omega, k_{x}, k_{y}, z\right)+\Gamma-\frac{k_{x}}{\Omega^{\prime}+i v_{\mathrm{k}} k_{\perp}^{2}} \frac{d u_{0}}{d z}\right) \\
& \cdot W\left(\omega, k_{x}, k_{y}, z\right) .
\end{aligned}
$$

Here, $\rho_{00}=\rho_{0}(z=0), k_{\perp}^{2}=k_{x}^{2}+k_{y}^{2}, \Omega^{\prime}=\Omega-k_{x} u_{0}$, $W=\left(\rho_{0} / \rho_{00}\right)^{1 / 2} w$,

$$
\begin{gathered}
Z_{0}=\rho_{00} c_{\mathrm{s}}\left[\frac{\left(\Omega^{\prime 2}+i \nu k_{\perp}^{2} \Omega^{\prime}\right)-\omega_{g}^{2}}{\left(\Omega^{\prime 2}+i \nu k_{\perp}^{2} \Omega^{\prime}\right)-c_{\mathrm{s}}^{2} k_{\perp}^{2}}\right]^{\frac{1}{2}}, \\
K^{2}=\frac{\left[\left(\Omega^{\prime 2}+i \nu k_{\perp}^{2} \Omega^{\prime}\right)-\omega_{g}^{2}\right]\left[\left(\Omega^{\prime 2}+i \nu k_{\perp}^{2} \Omega^{\prime}\right)-c_{\mathrm{s}}^{2} k_{\perp}^{2}\right]}{\Omega^{\prime}\left(\Omega^{\prime}+i \nu k_{\perp}^{2}\right)}, \\
\chi=\Gamma-\frac{1}{2} \frac{d u_{0}}{d z} \frac{k_{x}}{\Omega^{\prime}+i \nu_{\mathrm{k}} k_{\perp}^{2}}+\frac{1}{2} \frac{d \ln Z_{0}}{d z}, \\
\Gamma=\frac{(2-\gamma) g}{2 c_{\mathrm{s}}^{2}}-\frac{1}{2 T} \frac{\partial T}{\partial z},
\end{gathered}
$$

$\omega_{g}$ is the Brunt-Vaissala frequency, $\Gamma$ is the Ekkard parameter (Gossard and Hooke, 1975), and $\gamma$ is the ratio of specific heats. Equation (4a) is independent from Eq. (4b). This is a nonlinear first-order equation of the type of Rikkati's equation for the function $\Phi\left(\Omega, k_{x}, k_{y}, z\right)$. It is natural to use a radiation condition as the boundary condition for Eq. (4a), assuming that, at high altitudes $\left(z>z_{\max }\right)$, the temperature of the atmosphere and the wind can be considered to be independent of height. Then we find the first boundary condition:

$$
\Phi\left(z_{\max }\right)=\sqrt{1-\frac{\chi^{2}}{K^{2}}}+i \frac{\chi}{K} .
$$

Using the solution of Eq. (4a), it is possible to find a relationship for the vertical component of velocity by the integration of Eq. (4b). The second boundary condition on the surface of the Earth is determined by the nature of the source:

$$
W\left(\Omega, k_{\perp}, 0\right)=W_{\text {source }}\left(\Omega, k_{\perp}\right)
$$

Some results of the numerical solution of the system (4) with boundary conditions (5) and (6) for the vertical component of the medium velocity are presented in Fig. 2 . The calculation was performed for the vertical profiles corresponding to Fig. 1, the wave period is equal to $100 \mathrm{~s}$, and the horizontal wavelength is equal to $100 \mathrm{~km}$. Disturbances in question have such parameters that $\Omega^{\prime} \neq 0$ and their rate of decay due to the viscosity and thermal conductivity is not important. The characteristic vertical scales of such waves are several tens of kilometers and exceed the height of the homogeneous atmosphere and ionosphere at the relevant altitudes.

\section{Variation in the Electronic Density and Total Electrons Content in the Ionosphere}

To define the plasma density variations, we use quasihydrodynamic equations for electrons and ions (Gershman, 
1974). We do not take into account ionization and recombination processes and we neglect plasma deviations from electroneutrality, electron and ion viscosities and nonlinear terms $\left(\vec{u}_{e, i} \nabla\right) \vec{u}_{e, i}$. In that case

$$
\begin{gathered}
\frac{\partial n}{\partial t}+\nabla\left(n \vec{u}_{e, i}\right)=0 \\
-n m_{e, i} \frac{\partial \vec{u}_{e, i}}{\partial t}-\nabla p_{e, i}+m_{e, i} n \vec{g}+q_{e, i} n\left(\vec{E}+\frac{1}{c}\left[\vec{u}_{e, i}, \vec{B}\right]\right) \\
=m_{e, i} v_{e n, i n}\left(\vec{u}_{e, i}-\vec{u}_{n}\right) n
\end{gathered}
$$

Here, $n$ is the density of charged particles, $\vec{B}$ is the magnetic field, $m_{e, i}$ are the electron and ion masses, $q_{e, i}$ are the electron and ion charges, $\vec{u}_{e, i}$ are the velocities of charged particles, $p_{e, i}=k_{B} n T_{e, i}$ are the electron and ion pressures, $k_{B}$ is the Boltzmann constant, $T_{e, i}$ are the electron and ion temperatures, $v_{e n, i n}$ are the collision frequencies of electrons and ions with neutral particles, $\vec{u}_{n}$ is the velocity of neutral particles, and $\vec{E}$ is the electric field. We assume all values in the wave depend only on the vertical co-ordinate $z$. We neglect also the gravitational force on the charged particles because $g \ll \omega_{H i} \Omega \lambda$, where $\omega_{H i}$ is the ion gyrofrequency, $\Omega$ is the frequency of the infrasonic wave and $\lambda$ is the typical vertical scale of the infrasonic wave. Under these assumptions, we obtain an equation for the plasma density from Eq. (7):

$$
\frac{\partial n}{\partial t}=-\frac{\partial}{\partial z}\left\{\frac{v_{i n}^{2}+\omega_{H i}^{2} \cos ^{2} \theta}{v_{i n}^{2}+\omega_{H i}^{2}}\left(n w-D_{a} \frac{\partial n}{\partial z}\right)\right\} .
$$

Here, $D_{a}=k_{B}\left(T_{e}+T_{i}\right)\left(m_{i} v_{i n}\right)^{-1}$ is the coefficient of ambipolar diffusion, $\theta$ is the angle between the magnetic field and the vertical coordinate, and $w$ is the vertical component of the velocity of the neutral particles in the infrasonic wave. For altitudes of about $100 \mathrm{~km}$, the ambipolar diffusion coefficient has an order $D_{a} \sim 10^{-4}-10^{-2}$ $\mathrm{km}^{2} \mathrm{~s}^{-1}$, so Eq. (8) can be simplified for perturbations with the range of periods that are considered in this paper $(T)$, as $T \ll \lambda^{2} D_{a}^{-1}$. Let us assume that the velocity of neutral particles in the infrasonic wave changes as

$$
w(z, t)=A(z) \sin (\Omega t-k x),
$$

where $A(z)$ is the amplitude of the infrasonic wave. Taking into account a weak perturbation of the plasma density $n=$ $n_{0}+n_{\sim},\left|n_{\sim} / n_{0}\right| \ll 1$, from Eq. (8) we obtain:

$$
n_{\sim}=\frac{\partial}{\partial z}\left(\frac{v_{i n}^{2}+\omega_{H i}^{2} \cos ^{2} \theta}{v_{i n}^{2}+\omega_{H i}^{2}} n_{0} A(z)\right) \cos (\Omega t-k x) .
$$

The solution presented in Eq. (9) shows that the variation of the plasma density becomes large in regions with large gradients of undisturbed density $n_{0}$. Let us note that the infrasonic waves, considered in this paper, modulate not only the local, but also the total content of electronic density in the lower region of the ionosphere. Variation of the plasma density causes a modification of the reflection coefficient of VLF waves from the ionosphere $R$, and its rate of decay in the magnetospheric resonator.

\section{Whistler Waves: Rate of Decay in the Magne- tospheric Resonator}

The rate of decay of whistler waves in the magnetospheric resonator is determined by many factors, such as refraction, local damping and damping in the ionosphere. There is an uncertainty in the estimation of the first two factors due to ray-tracing problems. Here, we will examine damping in the ionosphere as the basic loss mechanism.

\subsection{Reflection coefficient of whistler mode waves nor-} mally incident on the ionosphere

Important preliminary results about the reflection coefficient were obtained analytically by Tverskoy (1968) and numerically by Tsuruda (1973) based on computing the signal at the Earth's surface.

In this paper, we analyze analytical expressions for the reflection coefficient of whistler mode waves with frequency $\omega$ and wave number $\kappa$ which are normally incident on the ionosphere from above. The form of these expressions makes it relatively simple to take into account the effect of different ionospheric factors, e.g., acoustic-gravity waves.

\subsection{Model problem statement and initial equations}

We now seek to find the reflection coefficient of a whistler wave. The ionospheric plasma is slightly inhomogeneous above $z=l$. The model altitude profile of the electron plasma density is presented in Fig. 3. Therefore, the approximation of geometric optics can be used to describe a whistler wave field in this region:

$$
\begin{gathered}
E_{x}=\frac{1}{\sqrt{|\kappa|\left(1+|P|^{2}\right)}}\left\{E_{1} \exp \left(i \int_{0}^{z} \kappa\left(z^{\prime}\right) d z^{\prime}\right)\right. \\
\left.+E_{2} \exp \left(-i \int_{0}^{z} \kappa\left(z^{\prime}\right) d z^{\prime}\right)\right\}, \\
E_{y}=P E_{z},
\end{gathered}
$$

where $E_{x}$ and $E_{y}$ are the horizontal electric fields in the whistler waves. In the range of whistler mode waves $\omega^{2} \ll$ $\omega_{p}^{2}, \omega_{B}^{2} \cos ^{2} \theta$, the polarization $P=i$ and the dispersion equation has the following form:

$$
\begin{aligned}
\frac{\kappa^{2} c^{2}}{\omega^{2}} \cong & \frac{\omega_{p}^{2} \omega_{B}}{\omega\left(\omega_{B}^{2}+v_{e n}^{2}\right)|\cos \theta|} \\
& \cdot\left\{1+\frac{i v_{e n}}{2 \omega_{B}|\cos \theta|}\left(1+\frac{\omega_{B}^{2}+v_{e n}^{2} \cos ^{2} \theta}{\omega_{B}^{2}+v_{e n}^{2}}\right)\right\},
\end{aligned}
$$

where $c$ is the speed of light, $\omega_{p}$ and $\omega_{B}$ are the electron plasma and cyclotron frequencies, and $v_{e n}$ is the electron collision frequency with neutral particles. We assume that $z=l$ is the coordinate of the boundary separating the region of applicability of geometrical optics. Then, the reflection coefficient of whistler-mode waves from the ionosphere can be determined with respect to the energy $R$ from a formula known in electrodynamics (Ginzburg, 1970):

$$
R=R^{\prime} \exp \left(-4 \operatorname{Im} \int_{l}^{\infty} \kappa\left(z^{\prime}\right) d z^{\prime}\right) .
$$


Taking into account that the ratio $v_{e n} / \omega_{B}$ is small, we obtain, from this formula, the expression:

$$
|\ln R|=\left|\ln R^{\prime}\right|+\frac{2 \sqrt{\omega}}{c\left(\sqrt{\omega_{B} \cos \theta}\right)^{3}} \int_{l}^{\infty} \omega_{p} v_{e n} d z
$$

Thus, our problem is reduced to computing the $\left|\ln R^{\prime}\right|$ value from the lower edge of the ionosphere.

At higher altitudes, the electron density changes rather sharply, decreasing by a factor of $e$ on scales of about 4 and $15 \mathrm{~km}$ under night-time and day-time conditions, respectively. Moreover, we should bear in mind that the whistlermode wavelength increases with decreasing plasma density. The approximation of geometric optics can be inapplicable in this case, and it is necessary to use a solution of the wave equation. For this purpose, we approximate the dependence of the plasma density by the exponential function (Bespalov and Mizonova, 2004):

$$
n(z)=n(z=l) \exp \left((z-l) / h_{i}\right)
$$

where $h_{i}$ is the characteristic altitude scale of the plasmadensity profile in the ionosphere.

We assume that the collision frequency of electrons with neutrals $v_{e n}$ does not change significantly. Then, the solution to the wave equation:

$$
\frac{d^{2} E_{x}}{d z^{2}}+\kappa^{2}(\omega, z) E_{x}=0
$$

is the function:

$$
E_{x}=E_{0}\left\{J_{0}\left[2 \kappa(\omega, z) h_{i}\right]+\beta N_{0}\left[2 \kappa(\omega, z) h_{i}\right]\right\},
$$

where $J_{0}$ and $N_{0}$ are Bessel functions, and $z_{*}$ is determined by the condition $\omega=\omega_{p}\left(z_{*}\right)$. The parameter $\beta$ is equal to

$$
\beta=-\frac{J_{0}\left(2 \omega h_{i} / c\right)+J_{1}\left(2 \omega h_{i} / c\right) \tan \left(\omega z_{*} / c\right)}{N_{0}\left(2 \omega h_{i} / c\right)+N_{1}\left(2 \omega h_{i} / c\right) \tan \left(\omega z_{*} / c\right)}
$$

for $z \leq z_{*}$ the refractive index is close to unity. Note that under the condition $\omega \ll c / h$, the parameter $\beta$ is close to zero. Now we can obtain the following expression for the reflection coefficient:

$$
R^{\prime}=\left[\frac{1+|\alpha|^{2}+2 \operatorname{Im} \alpha}{1+|\alpha|^{2}-2 \operatorname{Im} \alpha}\right]^{2}
$$

where:

$$
\alpha=\frac{J_{1}\left(2 \kappa_{l} h\right)+\beta N_{1}\left(2 \kappa_{l} h\right)}{J_{0}\left(2 \kappa_{l} h\right)+\beta N_{0}\left(2 \kappa_{l} h\right)}, \quad \kappa_{l}=\kappa(\omega, l)
$$

and hence:

$|\ln R|=2 \ln \frac{1+|\alpha|^{2}+2 \operatorname{Im} \alpha}{1+|\alpha|^{2}-2 \operatorname{Im} \alpha}+\frac{2 \sqrt{\omega}}{c\left(\sqrt{\omega_{B} \cos \theta}\right)^{3}} \int_{l}^{\infty} \omega_{p} v_{e n} d z$

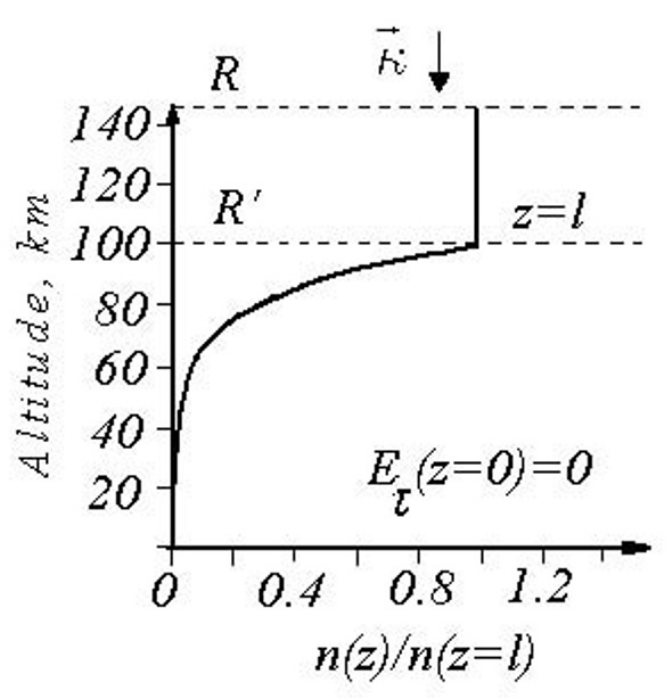

Fig. 3. The model altitude profile of the electron plasma density.

\subsection{Day and night conditions}

The variations in the logarithm of the reflection coefficients versus frequency under day-time and night-time conditions (with $h_{i}=15 \mathrm{~km}, n(z=l)=10^{5} \mathrm{~cm}^{-3}$ and $h_{i}=$ $4 \mathrm{~km}, n(z=l)=10^{4} \mathrm{~cm}^{-3}$ respectively) at different magnetic latitudes are illustrated in Fig. 4. The plots have been obtained from known experimental data on altitude profiles of charged particle density and collision frequency of electrons with neutrals (Gurevich and Shvartsburg, 1973).

Whistler-mode waves attenuate more effectively in the day-side ionosphere. This is related to the fact that the day-side lower boundary of the ionosphere is less sharp than the night-side boundary, and whistler-mode waves can penetrate to the region of intense attenuation where $v_{\text {en }} \sim$ $\omega_{B}$. Moreover, the ionospheric density of charged particles and the collision frequency of electrons with neutrals are higher under day-time conditions. Note that expressions for $|\ln R|$ and the corresponding figures have been obtained under the assumption that the conditions $h_{v_{e n}} \ll h_{i}, \kappa_{l} h_{i} \ll$ 1 and $c / h_{i} \leq \omega$ are satisfied.

The coefficient of reflection of whistler-mode waves from the ionosphere affects the $Q$-factor of the magnetospheric resonator. A detailed analysis (Bespalov and Trakhtengerts, 1986) indicates that the regime of stationary generation of whistler emissions occurs when the magnetospheric resonator quality is comparatively high (under night-time conditions), whereas the dynamic quasi-periodic regimes take place at a lower $Q$-factors (in the dawn and day-time magnetosphere).

In this case, for simplicity we will not consider the effects of refraction. Then, during the strictly longitudinal propagation the average decay of the whistler waves in the magnetospheric resonator is determined by the expression:

$$
v=\frac{2 \cdot|\ln R|}{T_{g}},
$$

where $R$ is the reflection coefficient from the ionosphere (Eq. (17)), and $T_{g}$ is the period of the group propagation of 

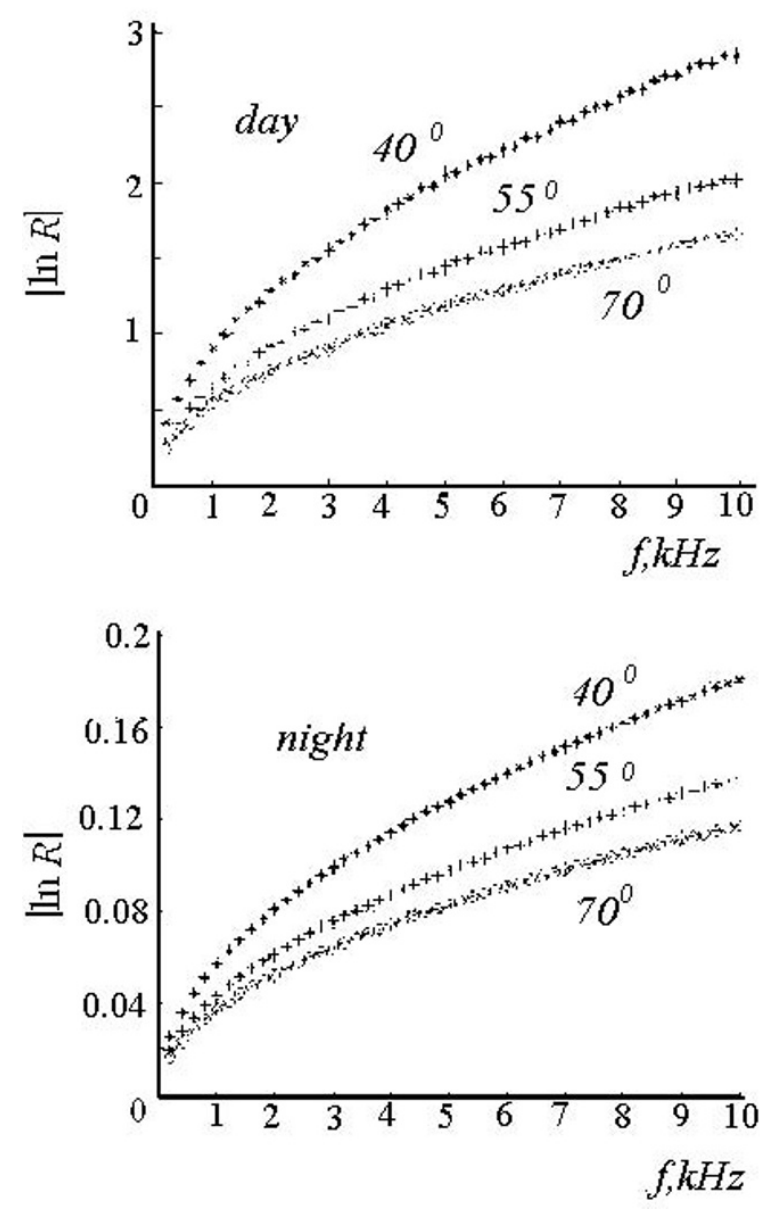

Fig. 4. Frequency dependence of the logarithm of the reflection coefficient under day-time and night-time conditions at different magnetic latitudes.

whistler waves in the magnetospheric resonator. If the reflection coefficient from one of the conjugated ionospheric regions contains a variable component connected with infrasound, then, as a consequence of Eqs. (9) and (17), it is possible to use the following expression for the whistlerwave decay rate:

$$
v=v_{0}\left(1+\mu \cos \left(\Omega t-k_{\psi} \psi\right)\right),
$$

where $\mu=n_{\sim} / n$, the wave number $k_{\psi}$ is connected with longitudinal structure of infrasonic waves, and $\psi$ is the azimuthal angle.

\section{Dynamics of Plasma Magnetospheric Maser}

The plasma magnetospheric maser is the semi-closed subsystem of the magnetosphere, whose dynamics are determined by the conditions for the interaction of waves and particles (see Fig. 5). The working conditions of this maser depend on the sources and sinks of particles and waves.

\subsection{Basic equations}

Previous research has shown that the cyclotron instability of a whistler wave in a separate magnetic flux tube in the electron radiation belts is similar to laboratory masers and lasers in many aspects (Bespalov and Trakhtengerts, 1986). In the plasma magnetospheric maser (PMM), a rather dense magnetized plasma, and conjugate areas of the ionosphere, form a "quasi-optical" resonator for whistler waves. The active particles are energetic electrons of the radiation belts. The role of the pump is carried out by sources of energetic electrons in the magnetic flux tube. There are several processes responsible for the formation of the particle source power, namely the diffusion of particles on magnetic shells, convection across magnetic field, and other factors.

Let us consider the importance of the case of the Earth's magnetosphere, when the power of the particle source is small and if the pitch-angle dependence is similar to the particle distribution function in a stationary state. Then slow processes (with temporal scales $\Delta t \gg T_{b}, T_{g}$; where $T_{b}$ is the period of bounce-oscillations of energetic electrons, and $T_{g}$ is the period of group propagation in a magnetospheric resonator) in the PMM are described by the following system of the balance equations (Bespalov and Trakhtengerts, 1986).

$$
\begin{aligned}
\frac{\partial N}{\partial t}+\Omega_{d} \frac{\partial N}{\partial \psi} & =-\delta \varepsilon N-\frac{N}{T}+J, \\
\frac{\partial \varepsilon}{\partial t} & =h \varepsilon N-v \varepsilon+a .
\end{aligned}
$$

Here, $N$ is the number of energetic electrons in a magnetic flux tube with unit cross-section at an ionospheric level, $\varepsilon$ is the energy density of whistler electromagnetic waves, averaged over a magnetic flux tube, $J$ is the source power of energetic electrons in the same magnetic flux tube, $T$ is the mean lifetime of energetic electrons in the magnetic trap excluding the influence of the cyclotron instability, $v$ is the whistler-wave rate of decay given by Eq. (19), and $a$ is the local power of other possible whistler electromagnetic waves sources connected, for example, with lightning discharges in the atmosphere. The interaction efficiency of waves and particles is determined by two coefficients: $\delta$ and $h$. Inside the plasmasphere, where the inequality $\beta_{*}=\left(\omega_{p L} v / \omega_{B L} c\right)^{2} \gg 1$ is fulfilled, they have the simple form:

$$
\delta=\frac{\omega_{B L}}{B_{L}^{2}}, \quad h=\frac{\omega_{B L}}{n_{p L} \sigma l},
$$

where $\omega_{B L}$ is the electronic cyclotron frequency and $B_{L}$ is the magnitude of the magnetic field in the flux tube at the equator, $\omega_{p L}^{2}=4 \pi n_{p L} e^{2} / m, n_{p L}$ is the density of cold plasma in the flux tube at the equator, $\sigma$ is the magnetic trap mirror ratio, and $l_{t}$ is the length of the magnetic trap. Averaged over the bounce-oscillation period of energetic electrons, an angular velocity of azimuth drift is defined by the expression (see Hamlin et al., 1961):

$$
\Omega_{d}=\frac{v^{2}}{\omega_{B L} r_{\circ}^{2} L^{2}}\left(1.05+0.45 \frac{v_{\perp L}}{v}\right)+\Omega_{\circ} \Theta\left(\frac{L_{p p}}{L}-1\right)
$$

where $v$ is the velocity of a particle, $r_{\circ}$ is the Earth's radius, $L$ is the parameter of the magnetic shell, $v_{\perp L}$ is the component of velocity of an electron at the magnetic equator perpendicular to the magnetic field, $\Omega_{\circ}$ is the Earth's angular velocity, $\Theta(y)$ is the unit function: $\Theta(y)=1$ if $y>0$ and $\Theta(y)=0$ if $y<0, L_{p p}$ is the plasmapause position. 


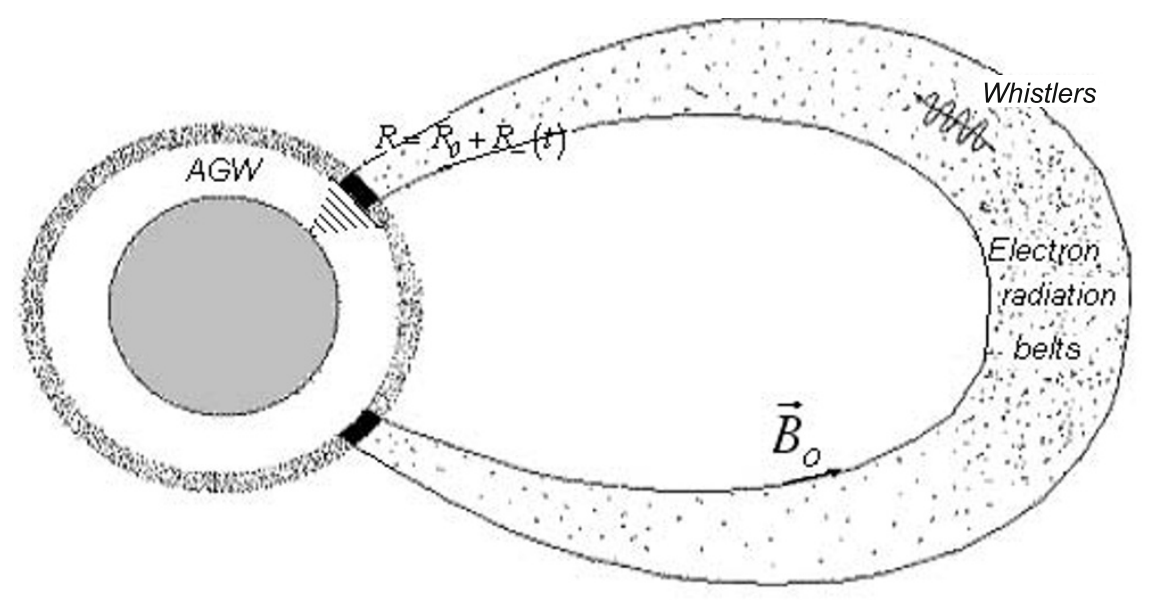

Fig. 5. PMM in the magnetic flux tube.

It follows from Eq. (20) that the parameters of the system can cause slowly decaying oscillations representing alternating stages of energetic electron accumulations and their precipitation into ionosphere during pulses of electromagnetic radiation. The near steady-state frequency of oscillations $\Omega_{J}$ and their decay $\nu_{J}$ are determined by Eq. (1):

$$
\Omega_{J}=\left(\frac{\omega_{B L} J}{n_{p} \sigma l_{t}}\right)^{1 / 2}, \quad v_{J}=\frac{\omega_{B L} J}{2 n_{p L} \sigma l_{t} \nu} .
$$

For typical conditions in the day-time sectors in the Earth's magnetosphere, the period of these oscillations $T_{J}=2 \pi / \Omega_{J}$ is between 10 and $150 \mathrm{~s}$, and the quality factor of the oscillations $Q_{J}=\Omega_{J} / 2 v_{J}$ in these regions is of the order of several tens. Such a high quality factor $Q_{J}$ defines the resonant response of the radiation belts to external effects. The system is ready for the generation of quasi-periodic VLF emissions, if there is a suitable external action.

In principle, it is possible to try to explain the strongest influences on the work of the PMM. For this purpose, it is necessary to assume that there are modulations of the power of the source of particles, the exterior wave source and the magnetospheric resonator quality. Additional calculations show that if the depth of modulation of these values is of a similar order, then the modulation of the magnetospheric resonator quality produces the strongest effects.

\subsection{Some results of numerical calculations}

Let us examine processes in the system of the balance Eq. (20), by considering that the damping decrease is determined by expression (19). Let us use dimensionless variables in the system of Eqs. (19) and (20):

$$
\begin{aligned}
& \tau=\Omega t, \tau_{\circ}=\Omega T_{\circ}, \omega_{d}=\Omega_{d} / \Omega, \nu_{*}=v / \Omega, \\
& j=h J / \Omega^{2}, \alpha=\delta a / \Omega^{2}, F=h N / \Omega, E=\delta \varepsilon / \Omega,
\end{aligned}
$$

where $\tau \gg 1, \tau_{\circ} \gg 1, \omega_{d} \ll 1, j \sim 1, v / \Omega \geq 1$.

From this, we obtain the system of equations:

$$
\begin{aligned}
& \frac{\partial F}{\partial \tau}=-E F-\frac{F}{\tau_{\circ}}+j(\psi)-\omega_{d} \frac{\partial F}{\partial \psi}, \\
& \frac{\partial E}{\partial \tau}=E F-\nu_{*}(\psi)\left[1+\mu \cos \left(\tau-\kappa_{\psi} \psi\right)\right] E+\alpha .
\end{aligned}
$$

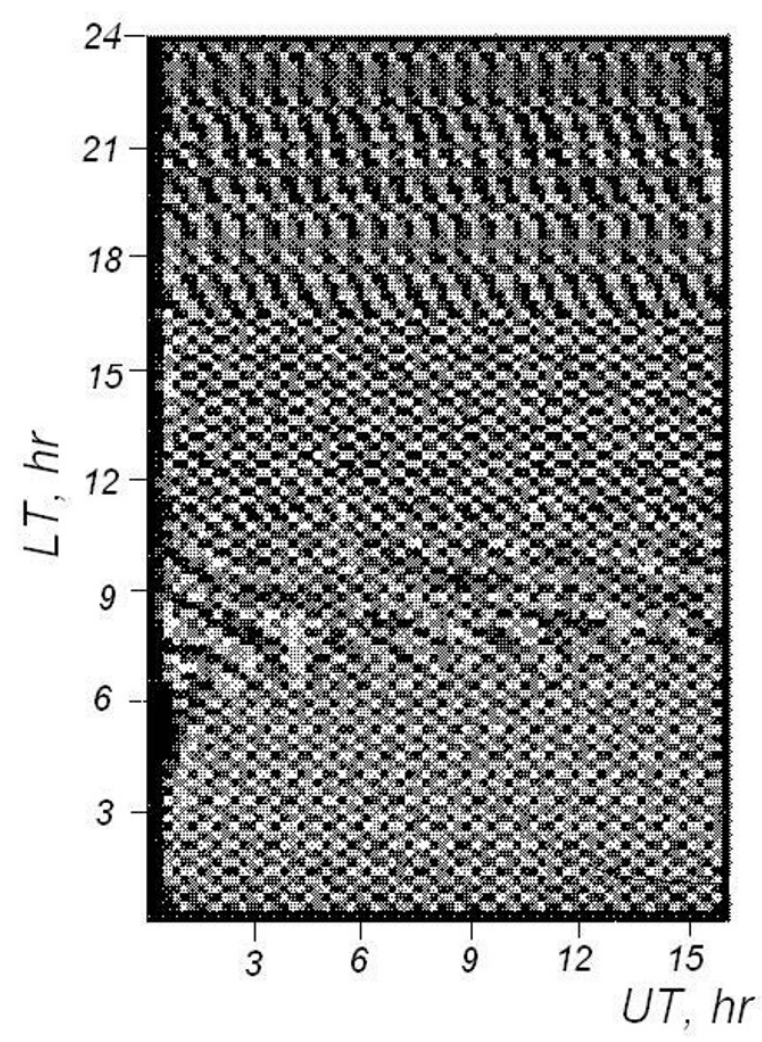

Fig. 6. Example of the time-spatial distribution of $E$ proportional to the whistler-waves energy density is presented by the level of grey. Calculations were carried out for model conditions (26).

Analysis of Eq. (25) was carried out numerically. Let the azimuthal angle $\psi$ be measured to the east from midnight. Typically, the particle source power has a maximum at dawn, and the mean whistler rate of decay has a maximum at noon. So we assume that

$j=1.5+0.5 \sin \psi, v_{*}=10-6 \cos \psi, \psi=(\pi / 12)(\mathrm{LT} / 1 \mathrm{hr})$,

$$
\tau_{\circ}=10^{3}, \omega_{d}=10^{-7}, \mu=0.03, a=0.005 .
$$

A typical example of the spatio-temporal distribution of 
the energy density of whistler waves $E$ is given in Fig. 6 to the domain LT, UT. Where LT is the local time in hours (LT is the space coordinate) and UT is the universal time in hours. The conditions of model calculation (26) accepted that the energetic electron power is stronger in the morning sector of the magnetosphere, and the whistler-waves rate of decay is greater in the day-time magnetosphere. The results of these calculations show that an infrasonic wave can form a spatio-temporal structure in the intensity distribution of the whistler emissions in the morning-side and day-side magnetosphere and can modulate fluxes of energetic electrons captured and precipitating into the ionosphere. Let us note that the modulation of the precipitated electron fluxes is considerably deeper than the modulation of captured electron fluxes.

\section{Conclusion}

Infrasound in the atmosphere is excited by different sources (Le Pichon et al., 2009), for example, by earthquakes, and by meteors, etc. In high-latitude regions, infrasound is excited also by moving auroral arcs.

Atmospheric infrasonic waves can influence the processes in electron radiation belts and the conditions for the generation of VLF emissions. Generation and modification of different types of quasi-periodic VLF emissions are possible. The following conditions are most favorable for such processes:

- the infrasonic wave must have a period between 30 and $150 \mathrm{~s}$ :

- the horizontal scale of the infrasonic wave must not be less than $100 \mathrm{~km}$;

- process can occur in the day-side magnetosphere;

- process can occur at sub-auroral latitudes.

We do not know of any publications in which experimental results about the influence of atmospheric infrasonic waves on the magnetospheric processes are discussed, and one of the basic purposes of this paper is draw attention of researchers to this possibility.

Acknowledgments. We are grateful to Prof. S. W. H. Cowley and Dr. R. Fear for help in the evolution of this work. This work was partly funded by ISSI 2006/2007 grant, by Program no. 22 of RAS, and by RFBR grant no. 12-02-00344.

\section{References}

Artru, J., P. Lognonne, and E. Blanc, Normal modes modelling of postseismic ionospheric oscillations, Geophys. Res. Lett., 28, 697-700, 2001.

Bespalov, P. A. and V. G. Mizonova, Reflection coefficient of whistler mode waves normally incident on the ionosphere, Geomagn. Aeron., 44, 49-53, 2004.

Bespalov, P. A. and O. N. Savina, Global synchronization of the fluctuations of the level of whistler emissions near Jupiter as the consequence of the three-dimensional rectification of the quality of the magnetospheric resonator, JETP Lett., 81, 151-155, 2005.

Bespalov, P. A. and V. Yu. Trakhtengerts, Dynamics of cyclotron instability in the Earth's radiation belts, in Rev. Plasma Phys., edited by Leontovich, M. A., 10, 155-292, Consultants Bureau, New York, London, 1986.

Bespalov, P. A., V. G. Mizonova, and O. N. Savina, Magnetospheric VLF response to the atmospheric infrasonic waves, Adv. Space Res., 31, 1235-1240, 2003.

Bespalov, P. A., O. N. Savina, and S. W. H. Cowley, Synchronized oscillations in whistler wave intensity and energetic electron fluxes in Jupiter's middle magnetosphere, J. Geophys. Res., 110, A09209, doi:10:1029/2005JA011147, 2005.

Gershman, B. N., Dynamics of Ionospheric Plasma, Nauka, Moscow, 1974 (in Russian).

Ginzburg, V. L., The Propagation of Electromagnetic Wave in Plasma, Pergamon, New York, 1970.

Gossard, E. and W. Hooke, Waves in the Atmosphere, Elsevier Scientific Publishing Company, Amsterdam-Oxford-New York, 1975.

Gurevich, A. V. and A. B. Shvartsburg, Nonlinear Theory of Radio Wave Propagation in the Ionosphere, Nauka, Moscow, 1973 (in Russian).

Hamlin, D. A., R. Karplus, R. C. Vik, and K. M. Watson, Mirror and azimuthal drift frequencies for geomagnetically trapped particles, $J$. Geophys. Res., 66, 1-4, 1961.

Le Pichon, A., E. Blanc, and A. Hauchecorne (eds.), Infrasound Monitoring for Atmospheric Studies, Hardcover, 2009.

Rapoport, V. O., P. A. Bespalov, N. A. Mityakov, M. Parrot, and N. A. Ryzhov, Feasibility study of ionospheric perturbations triggered by monochromatic infrasonic waves emitted with a ground-based experiment, J. Atmos. Sol.-Terr. Phys., 66, 1011-1017, 2004.

Savina, O. N., Acoustic-gravity waves in the atmosphere with a realistic temperature distribution, Geomagn. Aeron., 36, 218-224, 1996.

Savina, O. N., P. A. Bespalov, V. O. Rapoport, and N. A. Ryzhov, Generalized polarization relationships for acoustic gravity waves in the nonisothermic atmosphere with the wind, Geomagn. Aeron., 46, 247-253, 2006.

Tsuruda, K., Penetration and reflection of VLF waves through the ionosphere full wave calculations with ground effect, J. Atmos. Terr. Phys., 35, 1377-1405, 1973.

Tverskoy, B. A., Dynamics of Earth Radiation Belts, Nauka, Moscow, 1968 (in Russian).

P. A. Bespalov (e-mail: pbespalov@mail.ru) and O. N. Savina 